\title{
La investigación y el desarrollo como determinantes diferenciados y complementarios de la innovación y la productividad*
}

\author{
Andrés Barge-Gil \\ Alberto López \\ Universidad Complutense de Madrid
}

\section{Resumen}

La relación entre la inversión en I+D y los resultados innovadores y la productividad empresarial es uno de los temas más analizados por la economía de la innovación. Sin embargo, la mayoría de los trabajos no tienen en cuenta que la $I+D$ está formada por dos actividades diferentes: la investigación y el desarrollo. En este trabajo se analiza el efecto diferenciado que cada una de estas actividades tiene sobre los resultados innovadores, así como su efecto complementario sobre la productividad empresarial. Los resultados muestran que la investigación y el desarrollo tienen un efecto similar sobre la obtención de patentes, pero existen diferencias significativas al analizar otros resultados innovadores. En este sentido, el efecto de las actividades de desarrollo es mayor en la obtención de nuevos productos, así como para el éxito económico de los mismos. Finalmente, se encuentra evidencia a favor de un efecto complementario de la investigación y del desarrollo sobre la productividad empresarial.

Palabras clave: investigación, desarrollo, resultados innovadores, productividad, complementariedades

Clasificación JEL: D24, L25, O32, O33.

\begin{abstract}
The analysis of the relationship between $R \& D$ and innovation results and productivity has a long tradition in the literature on economics of innovation. However, a drawback of this literature is that $R \& D$ is mostly treated as a homogenous activity, while it includes two different activities: research and development. This article analyzes the differentiated effect of research and development on different innovation outputs, and tests the existence of complementarity between these activities in determining productivity. Our results show that the impact of research and development is similar when patent applications are analyzed, but we also find interesting differences when analyzing other innovation outputs. In this sense, the effect of development expenditures is much higher for the probability of introducing product innovations. Moreover, we find that development expenditures have a larger effect on sales from new products than research expenditures. Finally, we find evidence suggesting that there is complementarity between research and development in determining productivity.
\end{abstract}

Keywords: research, development, innovation results, productivity, complementarities

JEL classification: D24, L25, O32, O33.

* Los autores desean agradecer los comentarios realizados por un evaluador anónimo, así como la financiación recibida de la Fundación Ramón Areces. 


\section{Introducción}

Desde el trabajo seminal de Griliches (1979), un gran número de estudios empíricos se han centrado en analizar la relación entre I+D y productividad. Sirva como ejemplo de lo prolífico de esta literatura los numerosos trabajos que contienen una revisión de la misma (Griliches, 1995, y más recientemente Wieser, 2005, y Hall et al., 2010, contienen una revisión de esta literatura).

Por otra parte, existe otra rama de la literatura, muy relacionada con la anterior, que ha analizado los factores que determinan el éxito de las empresas a la hora de obtener resultados tecnológicos, ya sea en forma de introducción de innovaciones tecnológicas (Acs y Audretsch, 1987, 1988) o en forma de obtención de patentes (Scherer, 1983).

La conexión entre estas dos ramas de literatura empírica sobre innovación queda patente en el desarrollo posterior de modelos más complejos en los que la obtención de innovaciones (output de la innovación) por parte de las empresas depende de los gastos en innovación (input de la innovación) que realicen, y son estos outputs de la innovación los que, finalmente, tienen un impacto sobre la productividad. El trabajo de Crépon et al. (1998) es el pionero en proponer un modelo estructural (conocido como modelo $\mathrm{CDM}$ ) para analizar la relación entre gastos en $\mathrm{I}+\mathrm{D}$, innovación y productividad.

De toda la literatura anterior se desprende una conclusión clara: la I+D constituye la principal fuente de resultados innovadores y uno de los principales determinantes de los incrementos en la productividad de las empresas.

Sin embargo, en la literatura no se ha tenido en cuenta la posibilidad de que el impacto de la investigación (I) y el desarrollo (D) sea distinto. El análisis del efecto diferenciado de estas dos actividades es interesante y, posiblemente, necesario, ya que la investigación y el desarrollo son dos actividades intrínsecamente diferentes, cuyas diferencias se concretan en aspectos importantes, tales como sus objetivos, el tipo de conocimiento y el personal necesario para llevarlas a cabo, los estilos de gestión y el nivel de incertidumbre asociado. Una relación exhaustiva de las características que diferencian a investigación y desarrollo se puede encontrar en Barge-Gil y López (2015).

El objetivo de este trabajo es analizar los efectos diferenciados de la investigación y el desarrollo sobre dos tipos de resultados de las empresas: (i) los resultados innovadores, y (ii) la productividad. Los resultados que se presentan en este artículo constituyen un resumen de tres trabajos de investigación: Barge-Gil y López (2013a, 2013b y 2015).

El primero de los análisis se centra en el estudio del efecto diferenciado de la investigación y el desarrollo sobre tres resultados innovadores: la solicitud de patentes, la introducción de innovaciones tecnológicas (innovaciones de producto y de proceso) y las ventas innovadoras (ventas asociadas a productos nuevos introducidos por la empresa que son nuevos en el mercado en el que opera). Desde el punto de vista metodológico, en este análisis se presta especial atención al control de la 
simultaneidad de inputs y outputs, considerando la existencia de retardos en el efecto de la investigación y del desarrollo, que es una carencia detectada en estudios previos.

Por otra parte, en la relación entre investigación y desarrollo y productividad, nos centramos en la posible existencia de un efecto complementario de ambas actividades sobre la productividad (aunque también se realiza un análisis del efecto diferenciado de estas actividades). Por lo tanto, la metodología utilizada en este caso debe adecuarse a este objetivo principal: el punto de partida de la metodología utilizada consiste en elegir una función objetivo y testar la existencia de complementariedades a partir de la estimación de una medida de resultados en función de una combinación de prácticas.

Para realizar estos análisis, se utiliza, principalmente, la información que proporciona el Panel de Innovación Tecnológica (PITEC). Esta base de datos es un panel de empresas que contiene información sobre las actividades de innovación de las empresas españolas basada en los datos que proporciona la Encuesta sobre Innovación Tecnológica realizada por el INE. En particular, el PITEC proporciona información detallada de los gastos totales en $\mathrm{I}+\mathrm{D}$ de la empresa, permitiéndonos diferenciar entre gastos en investigación y gastos en desarrollo. La muestra de empresas utilizada en cada uno de los dos análisis realizados es diferente y se describirá en al apartado correspondiente.

El artículo está organizado del siguiente modo. El apartado 2 realiza una revisión breve de los estudios empíricos que se han centrado en analizar desde diferentes perspectivas y objetivos la investigación y el desarrollo como actividades diferenciadas. El apartado 3 analiza el efecto diferenciado de la investigación y el desarrollo sobre los resultados innovadores de las empresas. El apartado 4 se centra en analizar la existencia de un efecto complementario de las actividades de investigación y desarrollo sobre la productividad. Finalmente, el apartado 5 presenta las principales conclusiones.

\section{Estudios empíricos sobre las diferencias entre investigación y desarrollo}

Durante la década de los 80 algunos autores señalaron que la I+D incluye diferentes actividades y que el análisis de los determinantes y efectos de las mismas debería ser un tema central de los estudios sobre innovación (Mansfield, 1981; Link, 1982, 1985). Por ello, se llevaron a cabo estudios exploratorios con muchas limitaciones (sobre todo relacionadas con los datos y el tipo de análisis llevado a cabo), por lo que los propios autores advirtieron que sus resultados deberían interpretarse como muy preliminares.

Mansfield (1981) utiliza una encuesta de 108 empresas grandes de Estados Unidos para analizar los determinantes de la composición del gasto en I+D y los efectos de dicha composición sobre los resultados del proceso innovador. En este estudio se consideran cuatro tipos de gastos en I+D: (i) investigación básica, (ii) investigación en proyectos de largo plazo (al menos 5 años), (iii) investigación para obtener productos o procesos completamente nuevos y (iv) proyectos arriesgados (con menos 
del 50 por 100 de probabilidad de éxito). Uno de los principales resultados de este estudio es que las cuatro dimensiones no están demasiado relacionadas (cuando se compararan empresas pertenecientes al mismo sector). Además, este autor obtiene que las empresas grandes se hayan más orientadas a la investigación básica. Sin embargo, no se encuentra relación entre la concentración de mercado y el tipo de I+D. Por último, se obtiene cierta correlación positiva entre el número de innovaciones y la proporción de investigación básica.

Link (1982) analiza los determinantes de la investigación básica, investigación aplicada y desarrollo tecnológico para una muestra de 275 empresas que pertenecen a la US Fortune 100 list. Este autor obtiene que: (i) la orientación al desarrollo es más elevada para las empresas que operan en mercados muy concentrados y para las que reciben más financiación pública, (ii) las empresas con más beneficios presentan mayor orientación a la investigación aplicada, y (iii) la orientación a la investigación básica aumenta con la diversificación, con los beneficios (aunque en menor medida que la investigación aplicada) y con la gestión del propietario de la empresa.

En otro trabajo, Link (1985) adopta una perspectiva dinámica y obtiene que la orientación a la investigación básica y a la investigación de largo plazo decrece a lo largo del tiempo y analiza los determinantes de estos cambios para 146 empresas grandes de Estados Unidos. Este autor obtiene que la gestión estratégica de la empresa es un determinante muy importante. En este sentido, se obtiene que las empresas con una estrategia más ofensiva y con laboratorios de I+D se orientan en mayor medida a investigación básica y de largo plazo.

Sin embargo, a pesar de la relevancia de estos trabajos y de las recomendaciones de situar la heterogeneidad de la I+D en el centro del análisis, este tema no ha recibido apenas atención de los académicos, en parte debido a la ausencia de la información necesaria. Sin embargo, en los últimos años, y gracias a la disponibilidad de datos de las Encuestas Europeas de Innovación (Community Innovation Survey, CIS), algunos estudios han analizado la relación entre la financiación pública y la composición de la I+D (Aerts y Thorwarth, 2009; Clausen, 2009; Czarnitzki et al., 2011) ${ }^{1}$.

Aerts y Thorwarth (2009) usan una muestra de 521 empresas belgas de dos oleadas de la encuesta de I+D (2004 y 2006) y obtienen que existe adicionalidad ${ }^{2}$ de la financiación pública destinada a actividades de desarrollo pero no en la destinada a actividades de investigación.

Clausen (2009) usa una muestra de 1.019 empresas noruegas y distingue entre subsidios para actividades de investigación y subsidios para actividades de desarrollo. Este autor, a diferencia del trabajo anterior, encuentra que existe adicionalidad en los subsidios para actividades de investigación, pero no en los subsidios destinados a actividades de desarrollo.

\footnotetext{
1 En relación con los estudios teóricos, por ejemplo BANAL-ESTAÑOL y MACHO-STADLER (2010), presentan un modelo para analizar el efecto de los incentivos científicos y comerciales sobre la cantidad de tiempo empleada por los investigadores en actividades de desarrollo e investigación.

2 Entendiendo adicionalidad como incremento del gasto privado a consecuencia de la recepción de financiación pública.
} 
Czarnitzki et al. (2011) construyen un panel no balanceado desde 1999 hasta 2007 incluyendo 952 empresas belgas. Estos autores analizan las restricciones financieras asociadas con la I+D, obteniendo que el efecto negativo de estas restricciones es más elevado para las actividades de investigación.

Otra línea de investigación que ha renacido en los últimos años se centra en el análisis de los distintos determinantes de la investigación y el desarrollo. Por ejemplo, Barge-Gil y López (2014) analizan este tema utilizando datos de más de 20.000 observaciones del PITEC para el período 2005-2009. En este artículo se obtiene que la apropriabilidad y el tirón de la demanda tienen una influencia más significativa en los gastos de desarrollo, mientras que la oportunidad tecnológica tiene mayor influencia como determinante de los gastos de investigación.

Por último, y más relacionado con los resultados que se presentan en los Apartados 3 y 4, se puede citar el artículo de Czarnitzki et al. (2009). Estos autores analizan el impacto diferenciado de la investigación y el desarrollo sobre las patentes. Utilizan un panel no balanceado de 122 empresas desde 1993 hasta 2003 y obtienen que la relación entre patentes e I+D exhibe un premio para el peso de la investigación sobre el total de I+D. Sin embargo, debido al tamaño reducido de la muestra, Czarnitzki et al. (2009) consideran sus resultados como preliminares.

\section{El impacto diferenciado de la investigación y el desarrollo sobre los resultados innovadores}

El objetivo de este apartado es analizar el efecto diferenciado de la investigación y el desarrollo sobre tres resultados innovadores: la solicitud de patentes, la introducción de innovaciones tecnológicas (innovaciones de producto y de proceso) y las ventas innovadoras. En el estudio se utiliza una muestra de 4.024 empresas manufactureras del PITEC para el período 2005-2008. Este apartado es un resumen de la metodología y los resultados que se presentan en Barge-Gil y López (2015).

La organización del apartado es la siguiente. En primer lugar, en la sección 3.1 se describe la metodología utilizada para el estudio del efecto diferenciado de la investigación y el desarrollo sobre los resultados innovadores. En esta sección se describen las variables utilizadas y el método de estimación utilizado en cada uno de los análisis. En segundo lugar, la sección 3.2 presenta los principales resultados.

\subsection{Metodología: variables y método de estimación}

Este análisis parte de la utilización de una función de producción de conocimiento modificada, donde un resultado innovador $(R I)$ depende de los gastos en investigación y desarrollo y de un conjunto de variables de control $(Z)$ :

$$
R I_{J,(06-08)}=G\left(\delta+\alpha \text { IntensidadI }_{j,(05-07)}+\beta \text { IntensidadD }_{j,(05-07)}+\varphi Z_{j,(05-07)}+\varepsilon_{j}\right)
$$


$G$ representa una función lineal o no lineal (dependiendo de la variable dependiente considerada) y el subíndice $j$ hace referencia a la empresa.

Los resultados de la innovación en las encuestas tipo CIS se refieren a un período de tres años. Por ello, se consideran los resultados innovadores del período 2006-2008 (que se corresponden con las respuestas al cuestionario de 2008) como variable dependiente. Para definir las variables independientes se usa la información de los cuestionarios de 2005, 2006 y 2007. Por ejemplo, para obtener los gastos de innovación y los gastos de desarrollo se suman los gastos que reportan las empresas en los años 2005,2006 y $2007^{3}$. Usando esta metodología, se reducen los problemas de simultaneidad y endogeneidad que presentan otros análisis usando secciones cruzadas de alguna encuesta CIS (en muchos trabajos, los resultados innovadores de un período de tres años se explican utilizando como determinantes variables que hacen referencia exclusivamente al tercero de dichos años).

La definición de las variables dependientes es la siguiente. En el caso de las patentes se utilizan dos variables: (i) un indicador binario que toma el valor 1 si la empresa ha solicitado patentes y cero en caso contrario (variable Pat) y (ii) una variable cuantitativa que se define como el número de solicitudes de patentes por cada 100.000 empleados, en logaritmos (variable IntPat). Para las innovaciones de producto y de proceso se utilizan indicadores binarios que toman el valor 1 si la empresa ha obtenido nuevos procesos y nuevos productos, respectivamente (variables InnProd e InnProc). Por último, para las ventas innovadoras, se utilizan las ventas debidas a productos nuevos para el mercado por empleado, en logaritmos (variable VtsInn).

Como variables independientes, se distingue entre la intensidad de los gastos en investigación y de los gastos en desarrollo (variables IntensidadI e IntensidadD). La primera se define como lo gastos de investigación por empleado (en logaritmos) y la segunda como los gastos de desarrollo por empleado (en logaritmos).

Además, se controla por diversas características empresariales, denotadas por $Z$ en la expresión [3.1]. Estas variables de control son: el tamaño de la empresa, medida por el logaritmo de las ventas totales (variable Tamaño); la compra de I+D externa, medida como los gastos en I+D externa por empleado, en logaritmos (variable $I+D$ externa); la cooperación en innovación, medida como una variable ficticia que toma el valor 1 si la empresa coopera en innovación y cero en caso contrario (variable Cooperación); la importancia de los obstáculos a la innovación relacionados con los costes (variable Obstáculos coste) y con la información (variable Obstáculos información $)^{4}$; y la importancia de los «spillovers», medidos como la importancia

\footnotetext{
${ }^{3}$ Los resultados que se presentan en este trabajo son robustos a la consideración exclusivamente de los gastos en 2005 y 2006.

${ }^{4}$ Los obstáculos se valoran de 1 (muy importante) a 4 (no es un obstáculo). Se suman y reescalan en el intervalo 0-1. Los obstáculos asociados a los costes son: falta de fondos en la empresa o grupo; falta de fondos externos; costes de la innovación muy elevados. Los obstáculos asociados a la información son: falta de personal cualificado; falta de información sobre la tecnología; falta de información sobre el mercado, dificultad en encontrar socios para la innovación.
} 
de la información libremente disponible para la empresa (variable Spillovers) ${ }^{5}$. Por último, se consideran las diferencias entre sectores utilizando la clasificación de la OCDE de los distintos sectores económicos en función de su intensidad tecnológica (OCDE, 2005): media-baja, media-alta y alta, siendo el sector de baja tecnología la categoría de referencia (variables Media-baja tecnología; Media-alta tecnología; Alta tecnología).

Para el cálculo de las variables de intensidad de los gastos en investigación, intensidad de los gastos en desarrollo, intensidad de la gastos en I+D externa y tamaño, se usan medias ponderadas. Por ejemplo:

\section{IntensidadI $_{j,(05-07)}$}

$=\log \left(\frac{\text { gastos investigación }_{j, 05}+\text { gastos investigación }_{j, 06}+\text { gastos investigación }_{j, 07}}{\text { empleados }_{j, 05}+\text { empleados }_{j, 06}+\text { empleados }_{j, 07}}\right)$

Con respecto al método de estimación, para analizar el efecto diferenciado de la investigación y el desarrollo sobre las patentes se plantean las siguientes expresiones:

$$
\begin{aligned}
& \text { Pat }_{j,(06-08)} \\
& =G\left(\delta_{1}+\alpha_{1} \text { IntensidadI }_{j,(05-07)}+\beta_{1} \text { IntensidadD }_{j,(05-07)}+\varphi_{1} Z_{j,(05-07)}+\varepsilon_{1 j}\right) \\
& \text { IntPat }_{j,(06-08)} \\
& =G\left(\delta_{2}+\alpha_{2} \text { IntensidadI }_{j,(05-07)}+\beta_{2} \text { IntensidadD }_{j,(05-07)}+\varphi_{2} Z_{j,(05-07)}+\varepsilon_{1 j}\right)
\end{aligned}
$$

La expresión [3.2] se estima usando un modelo probit para toda la muestra y la expresión [3.3] utilizando MCO para la submuestra de empresas con patentes. La expresión [3.3] puede ser interpretada como la segunda ecuación de un modelo en dos partes $^{6}$. Al estimar ambas expresiones de forma separada, estamos permitiendo que diferentes mecanismos afecten a la probabilidad de patentar y a la intensidad con la que se patenta, por lo que los resultados de la expresión [3.3] no se pueden extrapolar a toda la población, sino solamente a las empresas que patentan. Como alternativa, se estima un modelo Tobit para la expresión [3.3] usando toda la muestra.

La obtención de innovaciones tecnológicas (innovaciones de producto y de proceso) se analiza a partir de las siguientes expresiones:

${ }^{5}$ Siguiendo a CASSIMAN y VEUGELERS (2002), definimos «spillovers» como las fuentes de información libremente disponibles, que se valoran de 1 (muy importante) a 4 (no es una fuente de información). Se suman y reescalan en el intervalo 0-1. Las fuentes de información son: conferencias, ferias comerciales y exposiciones; revistas científicas y publicaciones comerciales/técnicas; asociaciones profesionales y sectoriales.

${ }^{6}$ Para más discusión sobre este tema, véase WOOLDRIDGE (2002). 


$$
\begin{aligned}
& \text { InnProd }_{j,(06-08)} \\
& =G\left(\delta_{3}+\alpha_{3} \text { IntensidadI }_{j,(05-07)}+\beta_{3} \text { IntensidadD }_{j,(05-07)}+\varphi_{3} Z_{j,(05-07)}+\varepsilon_{3 j}\right) \\
& \text { InnProc }_{j,(06-08)} \\
& =G\left(\delta_{4}+\alpha_{4} \text { IntensidadI }_{j,(05-07)}+\beta_{4} \text { IntensidadD }_{j,(05-07)}+\varphi_{4} Z_{j,(05-07)}+\varepsilon_{4 j}\right)
\end{aligned}
$$

En primer lugar, las expresiones [3.4] y [3.5] se estiman de forma separada usando dos modelos probit. En segundo lugar, ambas expresiones se estiman de forma conjunta utilizando un probit bivariante.

Por último, la expresión a estimar en el caso de las ventas innovadoras es:

$$
\begin{aligned}
& V_{t s I n n}{ }_{j,(06-08)} \\
& =G\left(\delta_{5}+\alpha_{5} \text { IntensidadI }_{j,(05-07)}+\beta_{5} \text { IntensidadD }_{j,(05-07)}+\varphi_{5} Z_{j,(05-07)}+\varepsilon_{5 j}\right)
\end{aligned}
$$

En primer lugar, la expresión [3.6] se estima por MCO sobre la submuestra de empresas con innovaciones de producto. Esta expresión se podría interpretar como la segunda ecuación de un modelo en dos partes. En segundo lugar, se estima un modelo Tobit para el conjunto de todas las empresas de la muestra (tengan o no innovaciones de producto).

\subsection{Resultados}

El Cuadro 3.1 presenta los resultados del estudio de los efectos de la investigación y del desarrollo sobre la obtención de patentes. En este caso se obtiene que tanto los gastos en investigación como los gastos en desarrollo presentan un impacto positivo y significativo sobre la probabilidad de patentar. Además, cabe señalar que la magnitud del coeficiente es similar (véase la primera columna del Cuadro 3.1). Sin embargo, al analizar su efecto sobre el número de patentes de las empresas que patentan se observa que los coeficientes, aunque positivos, no resultan estadísticamente significativos (véase la segunda columna del Cuadro 3.1). Cuando ambos modelos se analizan conjuntamente, se restaura el efecto positivo y significativo de ambas variables que, de nuevo, presentan resultados muy similares (véase la tercera columna del Cuadro 3.1). 


\section{CUADRO 3.1}

\section{EFECTO DIFERENCIADO DE LA INVESTIGACIÓN Y EL DESARROLLO SOBRE LA OBTENCIÓN DE PATENTES}

\begin{tabular}{|c|c|c|c|c|c|c|}
\hline \multirow{3}{*}{\begin{tabular}{|l} 
\\
Intensidad I \\
\end{tabular}} & \multicolumn{4}{|c|}{ Modelo en dos partes } & \multirow{2}{*}{\multicolumn{2}{|c|}{$\begin{array}{c}\text { Tobit } \\
\text { (3) }\end{array}$}} \\
\hline & \multicolumn{2}{|c|}{$\begin{array}{c}(1) \\
\text { Probit }\end{array}$} & \multicolumn{2}{|c|}{$\begin{array}{c}\text { (2) } \\
\text { MCO }\end{array}$} & & \\
\hline & $0,011^{* * * *}$ & $(0,002)$ & 0,021 & $(0,013)$ & $0,575^{* * * *}$ & $(0,093)$ \\
\hline Intensidad D & $0,011^{* * * *}$ & $(0,002)$ & 0,016 & $(0,016)$ & $0,564^{* * * *}$ & $(0,115)$ \\
\hline Tamaño & $0,018^{* * *}$ & $(0,003)$ & $-0,541^{* * *}$ & $(0,029)$ & $0,673^{* * *}$ & $(0,165)$ \\
\hline$I+D$ externa & $0,011^{* * * *}$ & $(0,002)$ & 0,017 & $(0,013)$ & $0,567^{* * *}$ & $(0,089)$ \\
\hline Cooperación & $0,032^{* * *}$ & $(0,012)$ & $-0,168^{*}$ & $(0,088)$ & $1,467^{* *}$ & $(0,589)$ \\
\hline Obstáculos información & 0,014 & $(0,027)$ & 0,110 & $(0,191)$ & 0,689 & $(1,346)$ \\
\hline Obstáculos coste & $-0,007$ & $(0,022)$ & $-0,268^{*}$ & $(0,158)$ & $-0,448$ & $(1,091)$ \\
\hline Spillovers & $0,112^{* * * *}$ & $(0,021)$ & 0,034 & $(0,183)$ & $5,500^{* * * *}$ & $(1,034)$ \\
\hline Media-baja tecnología & $0,072^{* * *}$ & $(0,019)$ & 0,101 & $(0,123)$ & $3,406^{* * * *}$ & $(0,840)$ \\
\hline Media-alta tecnología & $0,086^{* * *}$ & $(0,017)$ & 0,121 & $(0,110)$ & $4,157^{* * * *}$ & $(0,773)$ \\
\hline Alta tecnología & $0,102^{* * * *}$ & $(0,025)$ & 0,170 & $(0,145)$ & $4,313^{* * * *}$ & $(0,953)$ \\
\hline Número de empresas & \multicolumn{2}{|c|}{4.024} & \multicolumn{2}{|c|}{647} & \multicolumn{2}{|c|}{4.024} \\
\hline $\mathrm{R}^{2}$ & \multicolumn{6}{|c|}{0,485} \\
\hline Log-Likelihood & \multicolumn{2}{|c|}{$-1.621,406$} & & & \multicolumn{2}{|c|}{$-3.530,199$} \\
\hline Pseudo $\mathrm{R}^{2}$ & \multicolumn{2}{|c|}{0,086} & & & \multicolumn{2}{|c|}{0,039} \\
\hline Test $I=D^{\mathrm{a}}$ & \multicolumn{2}{|c|}{0,921} & \multicolumn{2}{|c|}{0,805} & \multicolumn{2}{|c|}{0,934} \\
\hline
\end{tabular}

NOTAS: Coeficientes significativos al: 1 por $100^{* * *}, 5$ por $100 * *, 10$ por $100 *$. Errores estándar robustos a heterocedasticidad entre paréntesis. La estimación (1) muestra los efectos marginales de las variables independientes. Las variables dependientes son: En la estimación (1): Solicitud de patentes (variable ficticia que toma el valor 1 si la empresa solicitó patentes). En las estimaciones (2) y (3): Intensidad de solicitud de patentes (número de solicitudes por 100.000 empleados, en logaritmos). ${ }^{a}$ p-valor del test de igualdad de coeficientes de intensidad de I e intensidad de D.

Estos resultados difieren de los obtenidos por Czarnitzki et al. (2009), quienes obtienen que el peso de la investigación sobre el total de I+D afecta de manera positiva al número de patentes en una muestra de 122 empresas. Cabe señalar que, además de la diferencia en el tamaño muestral, el trabajo de Czarniztki et al. (2009) estudia los gastos en investigación y desarrollo contemporáneos a la solicitud de patentes (mientras que nosotros utilizamos los gastos pasados) y utiliza datos de panel, que permiten controlar por la heterogeneidad inobservable de las empresas. 
El segundo resultado innovador analizado es la obtención de innovaciones de producto y de proceso. En primer lugar, se estiman modelos probit separados para la innovación de producto y de proceso (véase la primera y segunda columna del Cuadro 3.2) y, en segundo lugar, se estima un modelo probit bivariante (véase la tercera y cuarta columna del Cuadro 3.3). Los resultados de ambas especificaciones son bastante similares y la hipótesis de que la correlación de los errores sea cero es rechazada, lo que sugiere que el modelo probit bivariante es más adecuado.

Se obtiene que tanto los gastos en investigación como en desarrollo presentan un efecto positivo y significativo tanto sobre la innovación de producto como sobre la innovación de proceso. La intensidad de los gastos en investigación tiene un efecto similar sobre ambas actividades. Sin embargo, la intensidad de los gastos en desarrollo presenta un efecto mucho mayor sobre la probabilidad de introducir nuevos productos que sobre la probabilidad de obtener nuevos procesos. Comparando el efecto de ambos tipos de gastos, el efecto de la intensidad de los gastos en desarrollo es un 50 por 100 mayor que el efecto de la intensidad de los gastos en investigación cuando los nuevos productos son analizados. Por el contrario, el efecto de la intensidad de los gastos en investigación es un 30 por 100 mayor que el efecto de la intensidad de los gastos en desarrollo cuando los nuevos procesos son analizados.

Para finalizar, el Cuadro 3 muestra los resultados referentes al tercer resultado innovador considerado: las ventas innovadoras. En primer lugar, se estima un modelo en dos partes para la probabilidad de introducir una innovación de producto y para la intensidad de ventas de productos innovadores en la submuestra de empresas innovadoras de producto (véase la primera y segunda columna del Cuadro 3.3). En segundo lugar, se estima un modelo Tobit para el total de la muestra (véase la tercera columna del Cuadro 3.3).

Los resultados de la primera parte del modelo en dos partes (primera columna del Cuadro 3.3) coinciden con lo estimado en el apartado anterior, mientras que la segunda parte de dicho modelo (segunda columna del Cuadro 3.3) muestra que tanto la intensidad de los gastos en desarrollo como la intensidad de los gastos en investigación tienen un efecto positivo y significativo sobre las ventas de productos innovadores. Sin embargo, existe una diferencia importante en la magnitud del efecto: el efecto de la intensidad de los gastos en desarrollo es un 44 por 100 superior al efecto de la intensidad de los gastos en investigación.

El modelo Tobit estima las dos partes conjuntamente para toda la muestra (tercera columna del Cuadro3.3). Sus resultados coinciden con los anteriores: ambos tipos de gasto tienen un efecto positivo y significativo sobre las ventas innovadoras, siendo el efecto de la intensidad de los gastos en desarrollo aproximadamente un 53 por 100 superior al de la intensidad de los gastos en investigación.

Estos resultados coinciden con la opinión mostrada por algunos autores que establece que la mayoría de innovaciones económicamente rentables no se basan en el conocimiento más reciente, sino en la aplicación de conocimiento ya existente (Kline y Rosenberg, 1986; Freeman, 1994; Klevorick et al., 1995). 


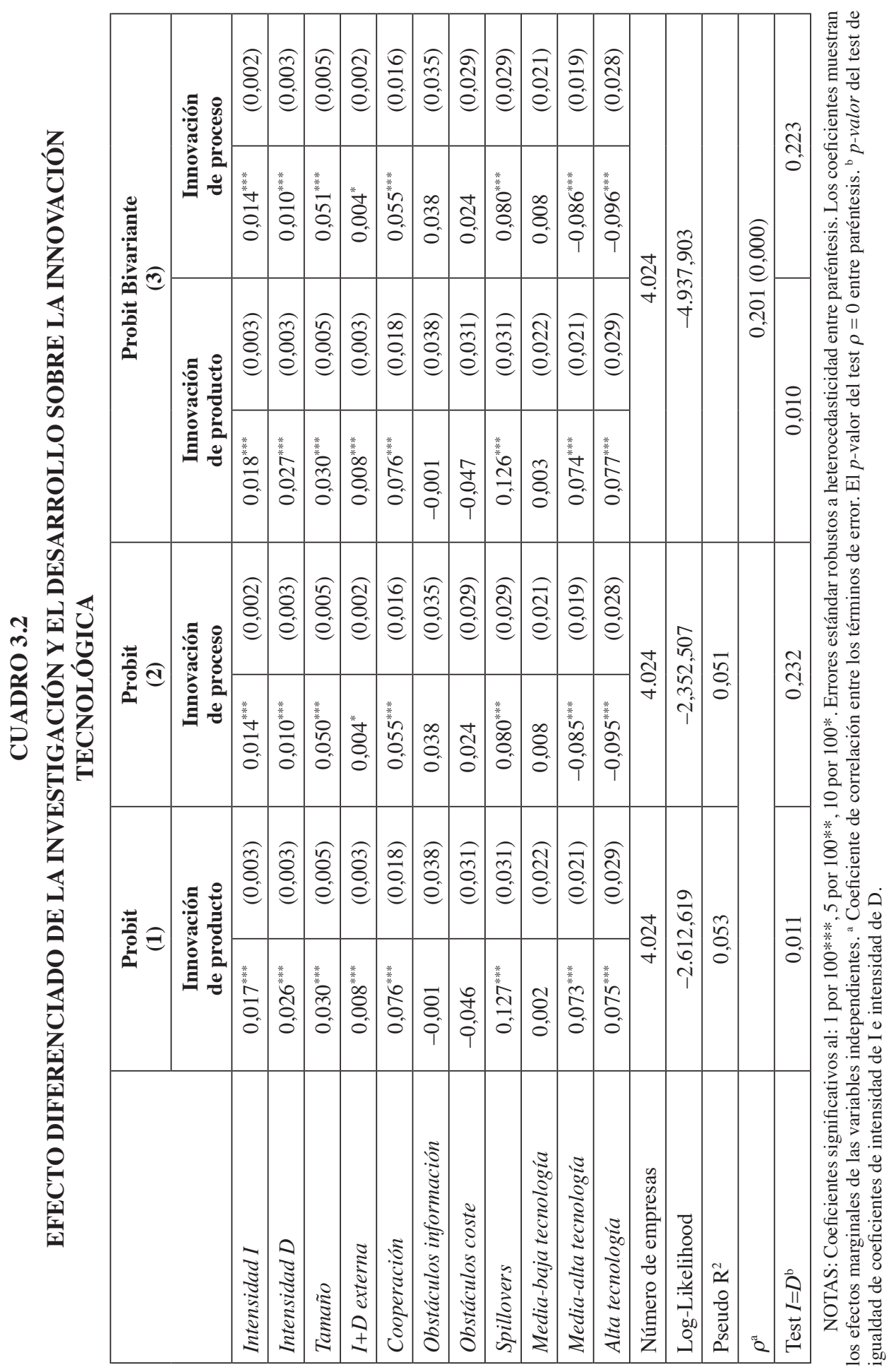


CUADRO 3.3

EFECTO DIFERENCIADO DE LA INVESTIGACIÓN Y EL DESARROLLO SOBRE LAS VENTAS INNOVADORAS

\begin{tabular}{|c|c|c|c|c|c|c|}
\hline \multirow[b]{3}{*}{ Intensidad I } & \multicolumn{4}{|c|}{ Modelo en dos partes } & \multirow{2}{*}{\multicolumn{2}{|c|}{$\begin{array}{c}\text { Tobit } \\
\text { (3) }\end{array}$}} \\
\hline & \multicolumn{2}{|c|}{$\begin{array}{c}\text { (1) } \\
\text { Probit }\end{array}$} & \multicolumn{2}{|c|}{$\begin{array}{c}(2) \\
\text { MCO }\end{array}$} & & \\
\hline & $0,017^{* * * *}$ & $(0,003)$ & $0,048^{* * *}$ & $(0,013)$ & $0,404^{* * * *}$ & $(0,057)$ \\
\hline Intensidad D & $0,026^{* * * *}$ & $(0,003)$ & $0,067^{* * *}$ & $(0,016)$ & $0,619^{\text {*****}}$ & $(0,071)$ \\
\hline Tamaño & $0,030^{* * * *}$ & $(0,005)$ & $0,153^{* * *}$ & $(0,025)$ & $0,723^{* * * *}$ & $(0,106)$ \\
\hline$I+D$ externa & $0,008^{* * *}$ & $(0,003)$ & 0,012 & $(0,012)$ & $0,186^{* * * *}$ & $(0,057)$ \\
\hline Cooperación & $0,076^{* * * *}$ & $(0,018)$ & $-0,094$ & $(0,081)$ & $1,560^{* * * *}$ & $(0,381)$ \\
\hline Obstáculos información & $-0,001$ & $(0,038)$ & $-0,026$ & $(0,175)$ & $-0,071$ & $(0,826)$ \\
\hline Obstáculos coste & $-0,046$ & $(0,031)$ & 0,218 & $(0,152)$ & $-0,867$ & $(0,681)$ \\
\hline Spillovers & $0,127^{* * * *}$ & $(0,031)$ & $-0,134$ & $(0,145)$ & $2,672^{* * * *}$ & $(0,681)$ \\
\hline Media-baja tecnología & 0,002 & $(0,022)$ & 0,074 & $(0,113)$ & 0,125 & $(0,512)$ \\
\hline Media-alta tecnología & $0,073^{* * * *}$ & $(0,021)$ & 0,129 & $(0,099)$ & $1,655^{\text {**** }}$ & $(0,454)$ \\
\hline Alta tecnología & $0,075^{* * * *}$ & $(0,029)$ & $-0,173$ & $(0,133)$ & $1,450^{* *}$ & $(0,601)$ \\
\hline Número de empresas & \multicolumn{2}{|c|}{4.024} & \multicolumn{2}{|c|}{1.770} & \multicolumn{2}{|c|}{4.024} \\
\hline $\mathrm{R}^{2}$ & & & \multicolumn{2}{|c|}{0,041} & & \\
\hline Log-Likelihood & \multicolumn{2}{|c|}{$-2.612,619$} & & & \multicolumn{2}{|c|}{$-8.024,669$} \\
\hline Pseudo $\mathrm{R}^{2}$ & \multicolumn{2}{|c|}{0,053} & & & \multicolumn{2}{|c|}{0,019} \\
\hline Test $I=D^{\mathrm{a}}$ & \multicolumn{2}{|c|}{0,011} & \multicolumn{2}{|c|}{0,294} & \multicolumn{2}{|c|}{0,008} \\
\hline
\end{tabular}

NOTAS: Coeficientes significativos al: 1 por $100 * * *, 5$ por $100 * *, 10$ por $100 *$. Errores estándar robustos a heterocedasticidad entre paréntesis. La estimación (1) muestra los efectos marginales de las variables independientes. Las variables dependientes son: En la estimación (1): Innovación de producto (variable ficticia que toma el valor igual a uno si la empresa declara haber introducido un bien o servicio nuevo en el mercado). En las estimaciones (2) y (3): Intensidad de ventas innovadoras (ratio entre ventas debidas a productos nuevos para el mercado y número de empleados, en logaritmos). ${ }^{a} p$-valor del test de igualdad de coeficientes de intensidad de I e intensidad de D.

\section{El efecto complementario de la investigación y el desarrollo sobre la productividad}

El objetivo principal de este apartado es analizar la existencia de un efecto complementario de las actividades de innovación y desarrollo sobre la productividad a nivel de empresas. El enfoque propuesto también permite estimar el efecto diferenciado de la investigación y del desarrollo en la productividad. Este apartado es un resumen de la metodología y los resultados que se presentan en Barge-Gil y López (2013a, 2013b). 
La organización del apartado es la siguiente. En primer lugar, en la sección 4.1 se describe la metodología utilizada para el estudio de la complementariedad entre investigación y desarrollo. En segundo lugar, la sección 4.2 se centra en la descripción de las bases de datos utilizadas y la muestra de empresas disponible. Finalmente, la sección 4.3 presenta los principales resultados.

\subsection{Enfoque empírico para el estudio de complementariedades entre la investigación y el desarrollo}

Dos actividades son complementarias si la aportación marginal de una de las actividades se incrementa a medida que aumenta la realización (o intensidad en la realización) de la otra actividad. El estudio de la existencia de complementariedades se basa en el concepto matemático de supermodularidad (véase Milgrom y Roberts, 1990) y puede ser abordado desde diferentes perspectivas (Athey y Stern, 1998, realizan una revisión de las diferentes metodologías existentes). En este estudio se utiliza el «enfoque directo» o «enfoque productividad». Los trabajos de Mohnen y Röller (2005) y Cassiman y Veugelers (2006) son dos ejemplos de uso de esta metodología.

Desde el punto de vista metodológico, el análisis parte de la estimación de una función de producción en la que se incorporan variables representativas de las actividades de investigación y desarrollo de las empresas, medidas tanto de forma discreta (mediante variables ficticias que representan la realización de estas actividades) como de forma continua (mediante los gastos en cada una de las actividades).

\section{Especificación de la función de producción}

Partimos de una función de producción Cobb-Douglas en la que, utilizando la especificación de Klette (1999), expresamos sus componentes en términos de desviaciones en logaritmos respecto de la media de la industria a la que pertenece cada empresa ${ }^{7}$.

$$
y_{j t}=a_{j t}+\beta_{k} k_{j t}+\beta_{l} l_{j t}+\beta_{m} m_{j t}+\varepsilon_{j t}
$$

Se utilizan letras minúsculas $(y, k, l$ y $m$ ) para indicar que las variables (producción, capital, trabajo y consumos intermedios) están medidas en términos de desviaciones en logaritmos respecto de la media de la industria (por ejemplo,

7 Esta especificación permite controlar por factores inobservados comunes a nivel de industria. Consideremos 47 industrias definidas aproximadamente a dos dígitos CNAE (una definición más detallada se encuentra en http://icono.fecyt.es/PITEC/Paginas/por_que.aspx). 
$y_{j t}=\ln \left(Y_{j t}\right)-\ln \left(\overline{Y_{i t}}\right)$, donde $\overline{Y_{i t}}$ es la producción media de las empresas en la industria $i$ en el año $t$, y el subíndice $j$ hace referencia a la empresa). El término $a_{j t}$ representa la productividad total de los factores y $\varepsilon_{j t}$ es un término de error de media cero y no correlacionado.

\section{Test de complementariedad utilizando variables de elección discretas}

En esta especificación se supone que el término de productividad depende de variables ficticias que indican si la empresa realiza actividades de investigación (Investigación ${ }_{j t}$ ) y de desarrollo (Desarrollo $o_{j t}$ ). También se incluye un término de heterogeneidad a nivel de empresa $\left(\eta_{j}\right)$ y de año $\left(\lambda_{t}\right)$.

$$
a_{j t}=\gamma_{i} \text { Investigación }_{j t}+\gamma_{d} \text { Desarrollo }_{j t}+\eta_{j}+\lambda_{t}
$$

Sustituyendo [4.2] en [4.1] se obtiene:

$$
y_{j t}=\beta_{k} k_{j t}+\beta_{l} l_{j t}+\beta_{m} m_{j t}+\gamma_{i} \text { Investigación }_{j t}+\gamma_{d} \text { Desarrollo }_{j t}+\eta_{j}+\lambda_{t}+\varepsilon_{j t}
$$

En el contexto de variables discretas, para realizar un test de complementariedad se tiene que obtener una restricción de desigualdad derivada de la teoría de la supermodularidad, y estudiar si esta restricción es aceptada por los datos. En concreto, hay que reescribir la expresión [4.3] incluyendo cuatro variables discretas mutuamente excluyentes que representan empresas con actividades de investigación y de desarrollo (Investigación\& Desarrollo ${ }_{j t}$ ), empresas solo con actividades de investigación (SoloInvestigación ${ }_{j t}$ ), empresas solo con actividades de desarrollo $\left(\right.$ SoloDesarrollo ${ }_{j t}$ ) y empresas sin actividades de investigación ni de desarrollo (Sin Investigación\&Desarrollo ${ }_{j t}$ ). Teniendo en cuenta estas cuatro variables, la expresión relevante es:

$$
\begin{aligned}
& y_{j t}=\beta_{k} k_{j t}+\beta_{l} l_{j t}+\beta_{m} m_{j t}+\gamma_{11} \text { Investigación\&Desarrollo }_{j t}+\gamma_{01} \text { SoloDesarrollo }_{j t}+ \\
& +\gamma_{10} \text { SoloInvestigación }_{j t}+\gamma_{00} \text { SinInvestigación\& Desarrollo }_{j t}+\eta_{j}+\lambda_{t}+\varepsilon_{j t}
\end{aligned}
$$

La investigación y el desarrollo son actividades complementarias si se cumple la siguiente restricción:

$$
\gamma_{11}-\gamma_{01}>\gamma_{10}-\gamma_{00}
$$

\section{Test de complementariedad utilizando variables continuas}

En este caso, suponemos que el término de productividad depende de variables continuas que representan los gastos en investigación $\left(\right.$ GastosI $_{j t}$ ) y en desarrollo 
$\left(\right.$ Gastos $\left._{i t}\right)$ de las empresas. Por consistencia con la especificación de la función de producción, los gastos en investigación y en desarrollo de cada empresa se expresan en términos de desviaciones en logaritmos respecto de la media de la industria a la que pertenece la empresa.

$$
a_{j t}=\delta_{i} \text { GastosI }_{j t}+\delta_{d} \operatorname{Gastos}_{j t}+\eta_{j}+\lambda_{t}
$$

Sustituyendo la expresión [4.6] en la expresión [4.1] se obtiene:

$$
y_{j t}=\beta_{k} k_{j t}+\beta_{l} l_{j t}+\beta_{m} m_{j t}+\delta_{i} \text { GastosI }_{j t}+\delta_{d} \operatorname{Gastos}_{j t}+\eta_{j}+\lambda_{t}+\varepsilon_{j t}
$$

En este contexto de variables continuas, la complementariedad entre gastos en investigación y en desarrollo se refleja en el término de interacción entre ambos tipos de gastos $\left(\right.$ GastosI $_{j t} *$ Gastos $\left._{j t}\right)$. Incluyendo este término de interacción en la expresión [4.7] se obtiene:

$$
\begin{aligned}
y_{j t}= & \beta_{k} k_{j t}+\beta_{l} l_{j t}+\beta_{m} m_{j t}+\delta_{i} \text { GastosI }_{j t}+\delta_{d} \text { Gastos }_{j t}+ \\
& +\delta_{i d}\left(\text { GastosI }_{j t} * \text { Gastos }_{j t}\right)+\eta_{j}+\lambda_{t}+\varepsilon_{j t}
\end{aligned}
$$

La obtención de una estimación positiva y significativa del coeficiente $\delta_{i d}$ implica la existencia de complementariedades entre los gastos en actividades de investigación y de desarrollo.

\subsection{Bases de datos y muestra de empresas}

Para este análisis ha sido necesario combinar la información del PITEC con información de la encuesta sobre el uso de TIC en las Empresas realizada por el INE. La razón es que la encuesta sobre el uso de TIC en las Empresas proporciona información sobre las compras de bienes y servicios (consumos intermedios) de las empresas. Esta información no está disponible en el PITEC y es necesaria para la estimación de una función de producción. El principal inconveniente derivado de la fusión de las encuestas utilizadas es que el análisis se restringe a una muestra de empresas grandes (empresas con 200 o más trabajadores). Esto se debe a las características de las bases de datos que se han utilizado y al conjunto de empresas disponible una vez realizada la fusión de las bases.

La muestra final utilizada está compuesta por 1.562 empresas grandes durante el período 2005-2009. La muestra incluye empresas de manufacturas y servicios, y se ha restringido a aquellas empresas que tienen al menos cuatro observaciones consecutivas. 


\subsection{Resultados}

Las diferentes especificaciones de la función de producción se estiman mediante system-GMM ${ }^{8}$. Los instrumentos utilizados en cada uno de los casos se detallan en los cuadros de resultados. También se proporcionan los resultados del test de Sargan de restricciones de sobreidentificación y los test de correlación serial de primer y segundo orden $\left(m_{1}\right.$ y $\left.m_{2}\right)$ propuestos por Arellano y Bond (1991).

En primer lugar, el Cuadro 4.1 muestra los resultados de la especificación de la función de producción utilizando variables ficticias para caracterizar la realización de actividades de investigación y de desarrollo. La primera columna presenta los resultados de la estimación sin tener en cuenta la posible existencia de complementariedades (estimación de la expresión [4.3]). Se obtiene que los coeficientes tanto de la investigación como del desarrollo son positivos y significativos. Además, la evidencia es a favor de un efecto similar de ambas actividades sobre la productividad.

La segunda columna del Cuadro 4.1 presenta los resultados del test de complementariedad entre las actividades de investigación y de desarrollo (estimación de la expresión [4.4]). Para analizar la complementariedad se realiza un test de una cola, en el que la hipótesis nula es $\mathrm{H}_{0}: \gamma_{11}-\gamma_{01} \leq \gamma_{10}-\gamma_{00}$, frente a la hipótesis alternativa $H_{a}: \gamma_{11}-\gamma_{01}>\gamma_{10}-\gamma_{00}$ (Cassiman y Veugelers, 2006, realizan un test similar). Se encuentra evidencia a favor de la existencia de complementariedades entre la realización de investigación y de desarrollo ( $\mathrm{p}$-valor $=0.039)$.

En segundo lugar, el Cuadro 4.2 presenta los resultados que se obtienen al utilizar las variables que representan los gastos de investigación y desarrollo de las empresas. La primera columna presenta los resultados sin tener en cuenta el factor de interacción entre ambos tipos de gastos (estimación de la expresión [4.7]). Se obtiene que ambos tipos de gastos tienen un efecto positivo y significativo, y de nuevo, dicho efecto es similar en magnitud.

La segunda columna del Cuadro 4.2 presenta los resultados teniendo en cuenta la interacción entre los gastos de investigación y de desarrollo (estimación de la expresión [4.8]). Los coeficientes de ambos tipos de gasto vuelven a ser positivos y significativos, aunque el resultado más importante de esta especificación es que el coeficiente que acompaña al término de interacción es positivo y significativo, lo que significa que de nuevo se encuentra evidencia a favor de la existencia de complementariedades entre la investigación y el desarrollo.

Para finalizar, cabe destacar que a lo largo de todos los resultados presentados, las estimaciones de los coeficientes de los factores productivos (capital, trabajo y consumos intermedios) son plausibles y coherentes con la literatura empírica sobre

8 Este método de estimación consiste en utilizar un estimador del método generalizado de los momentos que combina en un sistema la regresión de ecuaciones en primeras diferencias (con retardos en niveles de las variables como instrumentos) y en niveles (con retardos de los incrementos de las variables como instrumentos). BLUNDELL y BOND (1998) proporcionan una descripción de este método de estimación. 


\section{CUADRO 4.1 \\ I+D Y PRODUCTIVIDAD \\ TEST DE COMPLEMENTARIEDAD UTILIZANDO VARIABLES DE ELECCIÓN DISCRETAS}

\begin{tabular}{|c|c|c|c|c|}
\hline \multicolumn{5}{|l|}{$\begin{array}{l}\text { Período muestral: 2005-2009 } \\
\text { Número de empresas: } 1.562 \\
\text { Variable dependiente: } y\end{array}$} \\
\hline Variables explicativas & \multicolumn{2}{|c|}{ (1) } & \multicolumn{2}{|c|}{ (2) } \\
\hline$k$ & 0,062 & $(0,061)$ & 0,052 & $(0,053)$ \\
\hline$l$ & $0,473^{* * *}$ & $(0,134)$ & $0,498^{* * * *}$ & $(0,140)$ \\
\hline$m$ & $0,281^{* * *}$ & $(0,073)$ & $0,268^{* * * *}$ & $(0,119)$ \\
\hline Investigación & $0,054^{*}$ & $(0,030)$ & & \\
\hline Desarrollo & $0,054^{*}$ & $(0,032)$ & & \\
\hline Investigación\&Desarrollo & & & 0,079 & $(0,120)$ \\
\hline SoloDesarrollo & & & $-0,013$ & $(0,135)$ \\
\hline SoloInvestigación & & & $-0,027$ & $(0,138)$ \\
\hline SinInvestigación \&Desarrollo & & & $-0,038$ & $(0,140)$ \\
\hline Test de complementariedad, p-valor & & & \multicolumn{2}{|c|}{0,039} \\
\hline $\mathrm{m}_{1}$ (p-valor) & $-4,811$ & $(0,000)$ & $-4,048$ & $(0,000)$ \\
\hline $\mathrm{m}_{2}$ (p-valor) & $-0,014$ & $(0,988)$ & $-0,068$ & $(0,945)$ \\
\hline Test de Sargan, gl = 19 (p-valor) & 20,192 & $(0,383)$ & 18,279 & $(0,504)$ \\
\hline
\end{tabular}

NOTAS: Coeficientes significativos al: 1 por $100 * * *, 10$ por $100 *$. Errores estándar robustos a heterocedasticidad entre paréntesis. Las regresiones incluyen variables ficticias de año y una variable ficticia para manufacturas. Instrumentos para la ecuación en diferencias: retardo $\mathrm{t}-2$ en niveles de $k$; retardos $\mathrm{t}-2$ y $\mathrm{t}-3$ en niveles de $l$ y $m$. Instrumentos para la ecuación en niveles: retardo t-1 de las primeras diferencias de $k, l$ y $m$. El test de complementariedad es un test de una cola, en el que la hipótesis nula es $\mathrm{H}_{0}: \gamma_{11}-\gamma_{01} \leq \gamma_{10}-\gamma_{00}$ frente a la hipótesis alternativa $\mathrm{H}_{\mathrm{a}}: \gamma_{11}-\gamma_{01}>\gamma_{10}-\gamma_{00}$. Se muestra el $\mathrm{p}$-valor de este test.

estimación de funciones de producción ${ }^{9}$. Además, los resultados de los test de especificación (test de Sargan y de correlación serial) son satisfactorios.

En resumen, los resultados que se presentan en este apartado permiten obtener dos conclusiones. En primer lugar, tanto la investigación como el desarrollo tienen efectos significativos sobre la productividad de las empresas. Además, estos efectos son de magnitud similar. En segundo lugar, y lo que constituye el principal resultado, se ha obtenido evidencia a favor de la existencia de complementariedades entre las actividades de investigación y desarrollo de las empresas. Es decir, la realización conjunta de estas actividades tiene un efecto positivo sobre la productividad de las empresas. Sin embargo, quedan cuestiones importantes pendientes de tratar. En

9 La obtención de un coeficiente del capital bajo y no significativo es recurrente en la literatura de estimación de funciones de producción utilizando técnicas de GMM. GRILICHES y MAIRESSE (1998) analizan este tema). 
CUADRO 4.2

I+D Y PRODUCTIVIDAD

TEST DE COMPLEMENTARIEDAD UTILIZANDO VARIABLES CONTINUAS

\begin{tabular}{|l|l|l|l|c|}
\hline $\begin{array}{l}\text { Período muestral: } 2005-2009 \\
\text { Número de empresas: } 1.562 \\
\text { Variable dependiente: } y\end{array}$ & \multicolumn{2}{|c|}{$(\mathbf{1})$} & \multicolumn{3}{|c|}{$(\mathbf{2})$} \\
\hline Variables explicativas & 0,052 & $(0,058)$ & 0,053 & $(0,058)$ \\
\hline$k$ & $0,544^{* * *}$ & $(0,119)$ & $0,551^{* * *}$ & $(0,118)$ \\
\hline$l$ & $0,219^{* * *}$ & $(0,081)$ & $0,221^{* * *}$ & $(0,082)$ \\
\hline$m$ & $0,006^{* *}$ & $(0,003)$ & $0,008^{* *}$ & $(0,004)$ \\
\hline GastosI & $0,007^{*}$ & $(0,004)$ & $0,010^{* *}$ & $(0,005)$ \\
\hline GastosD & & & $0,0006^{*}$ & $(0,0003)$ \\
\hline GastosI*GastosD & $-4,613$ & $(0,000)$ & $-4,629$ & $(0,000)$ \\
\hline $\mathrm{m}_{1}$ (p-valor) & $-0,289$ & $(0,773)$ & $-0,241$ & $(0,809)$ \\
\hline $\mathrm{m}_{2}$ (p-valor) & 20,724 & $(0,352)$ & 20,965 & $(0,339)$ \\
\hline Test de Sargan, gl = 19 (p-valor) & & & & \\
\hline
\end{tabular}

NOTAS: Coeficientes significativos al: 1 por $100^{* * *}, 5$ por $100^{* *}, 10$ por 100*. Errores estándar robustos a heterocedasticidad entre paréntesis. Las regresiones incluyen variables ficticias de año y una variable ficticia para manufacturas. Instrumentos para la ecuación en diferencias: retardo t- 2 en niveles de $k$; retardos t-2 y t-3 en niveles de $l$ y $m$. Instrumentos para la ecuación en niveles: retardo t- 1 de las primeras diferencias de $k, l$ y $m$.

concreto, en este análisis no se ha tenido en cuenta la endogeneidad potencial de las actividades de investigación y desarrollo. Esta cuestión merece ser analiza en futuros trabajos.

\section{Conclusiones}

La inversión en I+D constituye el principal recurso para las actividades innovadoras y, por ello, su efecto sobre los resultados innovadores y la productividad ha sido objeto de numerosos estudios empíricos.

Sin embargo, los estudios previos casi nunca han tenido en cuenta que la I+D engloba dos actividades diferentes en objetivos, tipo de conocimiento y personal necesario para llevarlas a cabo, además de en los estilos de gestión y el nivel de incertidumbre asociado.

El objetivo de este artículo es contribuir al debate sobre la importancia de la I+D en los resultados empresariales y, en particular, introducir un nuevo elemento en este debate, centrándose el análisis en el papel diferenciado que estas actividades pueden tener. Para la consecución de este objetivo, este trabajo presenta la revisión de una serie de trabajos recientes que se han centrado en analizar los efectos diferenciados 
de la investigación y el desarrollo sobre dos tipos de resultados de las empresas: (i) los resultados innovadores, y (ii) la productividad. Una característica común de estos trabajos es la utilización como fuente de información del Panel de Innovación Tecnológica (PITEC). En este contexto, la característica fundamental del PITEC, y sin la cual no se podrían realizar los análisis que se presentan, es que permite diferenciar entre gastos en investigación y gastos en desarrollo de las empresas.

El primer conjunto de resultados se centra en el análisis de los efectos diferenciados de la investigación y del desarrollo sobre tres tipos de resultados innovadores: la solicitud de patentes, la introducción de innovaciones tecnológicas (innovaciones de producto y de proceso) y las ventas innovadoras (ventas asociadas a productos nuevos introducidos por la empresa que son nuevos en el mercado en el que opera). La principal conclusión de este análisis es que, si bien tanto la investigación como el desarrollo son importantes a la hora de obtener resultados innovadores, existen diferencias significativas entre los efectos de ambas actividades. En primer lugar, la investigación y el desarrollo tienen un efecto positivo y de magnitud similar sobre la solicitud de patentes. En segundo lugar, las actividades de investigación tienen un mayor efecto (positivo) que las actividades de desarrollo sobre la obtención de innovaciones de proceso. En tercer lugar, las actividades de desarrollo tienen un mayor efecto (positivo) que las actividades de investigación sobre la obtención de innovaciones de producto, siendo especialmente importante esta diferencia cuando se analizan las ventas debidas a nuevos productos.

El otro aspecto analizado en el artículo es el efecto de la I+D sobre la productividad. En este caso, el objetivo principal es analizar la existencia de un efecto complementario de las actividades de investigación y de desarrollo sobre la productividad (aunque también se realiza un análisis del efecto diferenciado de estas actividades). En este análisis se obtiene que tanto la investigación como el desarrollo tienen efectos positivos sobre la productividad empresarial. Sin embargo, la principal conclusión es que existe evidencia a favor de un efecto complementario de dichas actividades, es decir, la realización conjunta de actividades de investigación y de desarrollo contribuye a incrementar la productividad empresarial.

\section{Referencias bibliográficas}

[1] ACS, Z.J. y AUDRETSCH, D.B. (1987). «Innovation, market share, and firm size». Review of Economics and Statistics, 69(4), 567-575.

[2] ACS, Z.J. y AUDRETSCH, D.B. (1988). «Innovation in large and small firms: An empirical analysis». American Economic Review, 78(4), 678-690.

[3] AERTS, K. y THORWARTH, S. (2009). «Additionality effects of public R\&D funding: $\mathrm{R}$ vs. D». FBE Research Report MSI_0811, K.U.Leuven.

[4] ARELLANO, M. y BOND, S. (1991). «Some tests of specification for panel data: Monte Carlo evidence and an application to employment equations». Review of Economic Studies, 58, 277-297. 
[5] ATHEY S. y STERN S. (1998). «An empirical framework for testing theories about complementarity in organizational design». NBER Working Paper 6600.

[6] BANAL-ESTAÑOL, A. y MACHO-STADLER, I. (2010). «Scientific and commercial incentives in R\&D: Research versus development?». Journal of Economics \& Management Strategy, 19(1), 185-221.

[7] BARGE-GIL, A. y LÓPEZ, A. (2013a). «The complementarity effect of research and development on firm productivity». Applied Economics Letters, 20(15), 1426-1430.

[8] BARGE-GIL, A. y LÓPEZ, A. (2013b). «R\&D and productivity: In search of complementarity between research and development activities». MPRA Paper 43808.

[9] BARGE-GIL, A. y LÓPEZ, A. (2014). «R\&D determinants: accounting for the differences between research and development». Research Policy, 43(9), 1634-1648.

[10] BARGE-GIL, A. y LÓPEZ, A. (2015). «R versus D: Estimating the differentiated effect of research and development on innovation results». Industrial and Corporate Change, 24(1), 93-129.

[11] BLUNDELL, R. y BOND, S. (1998). «Initial conditions and moment restrictions in dynamic panel data models». Journal of Econometrics, 87, 115-143.

[12] CASSIMAN, B. y VEUGELERS, R. (2002). «R\&D cooperation and spillovers: some empirical evidence from Belgium». American Economic Review, 92 (4), 1169-1184.

[13] CASSIMAN, B. y VEUGELERS, R. (2006). "In search of complementarity in innovation strategy: Internal R\&D and external knowledge acquisition». Management Science, 52(1), 68-82.

[14] CLAUSEN, T.H. (2009). «Do subsidies have positive impacts on R\&D and innovation activities at the firm level?». Structural Change and Economic Dynamics, 20, 239-253.

[15] CREPON, B.; DUGUET, E. y MAIRESSE, J. (1998). «Research, innovation and productivity: An econometric analysis at the firm level». Economics of Innovation and New Technology, 7, 115-158.

[16] CZARNITZKI, D., KRAFT, K. y THORWARTH, S. (2009). «The knowledge production of "R" and "D", Economic Letters, 105, 141-143.

[17] CZARNITZKi, D.,HOTTENROTT, H. y THORWARTH, S. (2011). «Industrial research versus development investment: the implications of financial constraints». Cambridge Journal of Economics 35(3), 527-544.

[18] FREEMAN, C. (1994). «The economics of technical change». Cambridge Journal of Economics, 18, 463-514.

[19] GRILICHES, Z. (1979). «Issues in assessing the contribution of research and development to productivity growth». Bell Journal of Economics, 10(1), 92-116.

[20] GRILICHES, Z. (1995). «R\&D and productivity: Econometric results and measurement issues». en Handbook of the economics of innovation and technological change, ed. P. Stoneman. Oxford, U.K., and Cambridge, Mass.: Basil Blackwell, 52-89.

[21] GRILICHES, Z. y MAIRESSE, J. (1998). «Production functions: The search for identification». en Steinar Strøm, ed., Econometrics and Economic Theory in the 20th Century: the Ragnar Frisch Centennial Symposium, Cambridge University Press: Cambridge, UK, 169-203.

[22] HALL, B.H., MAIRESSE, J. y MOHNEN, P. (2010). «Measuring the returns to R\&D». en B.H. Hall y N. Rosenberg (eds.), Handbook of the Economics of Innovation.

[23] KLETTE, T.J. (1999). «Market power, scale economies and productivity: estimates from a panel of establishment data». Journal of Industrial Economics, 48, 451-476. 
[24] KLEVORICK, A., LEVIN, R., NELSON, R. y WINTER, S. (1995). «On the sources and significance of inter-industry differences in technological opportunities». Research Policy, 24, 185-205.

[25] KLINE, S. y ROSENBERG, N. (1986). «An overview of innovation», en R. Landau y N. Rosenberg (eds.), The Positive Sum Strategy: Harnessing Technology for Economic Growth. National Academic Press: Washington DC, 273-305.

[26] LINK, A.N. (1982). «An analysis of the composition of R\&D spending». Southern Economic Journal, 49(2), 342-349.

[27] LINK, A.N. (1985). «The changing composition of R\&D». Managerial and Decision Economics, 6(2), 125-128.

[28] MANSFIELD, E. (1981). «Composition of R and D expenditures: Relationship to size of firm, concentration and innovative output». Review of Economics and Statistics, 63(4), 610-615.

[29] MILGROM, P. y ROBERTS, J. (1990). «The economics of modern manufacturing: Technology, strategy, and organization». American Economic Review, 80(3), 511-528.

[30] MOHNEN, P. y RÖLLER, L.H. (2005). «Complementarities in innovation policy». European Economic Review, 49(6), 1431-1450.

[31] OCDE (2005). Oslo Manual. Guidelines for collecting and interpreting innovation-3rd Edition. OECD Publications: Paris.

[32] SCHERER, F.M. (1983). «The propensity to patent». International Journal of Industrial Organization, 1, 107-28.

[33] WIESER, R. (2005). «Research and development, productivity and spillovers: Empirical evidence at the firm level». Journal of Economic Surveys, 19(4), 587-621.

[34] WOOLDRIDGE, J. (2002). Econometric Analysis of Cross Section and Panel Data. MIT Press. 